\title{
Pé diabético: avaliação dos fatores de risco relacionados a amputações maiores e menores
}

\author{
Pé diabético: avaliação dos fatores de risco relacionados a amputações maiores e \\ menores
}

\begin{abstract}
Pie diabético: evaluación de factores de riesgo relacionados con amputaciones mayores y menores
\end{abstract}

Janeide Pereira dos Santos de Gois ${ }^{1 *}$, Arlane Silva Carvalho Chaves ${ }^{1}$.

\section{RESUMO}

Objetivo: Identificar os fatores de risco que contribuíram para amputação maior e menor em pacientes com pé diabético no Hospital Municipal de Imperatriz. Métodos: Trata de um estudo analítico de abordagem quantitativa. Foi utilizado um questionário contendo variáveis sociodemográficas, aspectos socioeconômicos e aspectos clínicos. A amostra foi de 40 pacientes com pé diabético que sofreram tratamento cirúrgico. Resultados: Dos entrevistados 55\% foi sexo feminino. Com idade entre 38 a $93(65,08 \%)$ anos. Com renda de até dois salários mínimos $(87,5 \%)$, procedentes de Imperatriz $(32,5 \%)$. Como fatores de risco para amputação: $65 \%$ eram tabagistas, $42,5 \%$ etilista e $65 \%$ tinha história de diabetes mellitus na família. Quanto as comorbidades $57,5 \%$ tinha hipertensão arterial sistêmica, 52,5\% dislipidemia e $27,5 \%$ doença coronariana. Sobre os cuidados e orientação: $32,5 \%$ não tinham o hábito de caminhar descalço, $72,5 \%$ nunca tiveram seus pés examinados por profissional de saúde e $87,5 \%$ nunca receberam orientação de cuidados por profissional de saúde. Conclusão: A maioria dos fatores que colaboram para a amputação de pé diabético, podem ser evitados ou controlados com medidas de prevenção, tais como cuidados simples e orientação para o autocuidado, de modo que corrobore para evitar o aparecimento do pé diabético e/ou complicações, sobretudo das amputações.

Palavras-Chave: Pé diabético, Fatores de risco, Amputação.

\begin{abstract}
Objective: To identify the risk factors that contributed to major and minor amputation in patients with diabetic foot at the Municipal Hospital of Imperatriz. Methods: This is an analytical study of quantitative approach. A questionnaire containing sociodemographic variables, socioeconomic aspects and clinical aspects was used. The sample consisted of 40 patients with diabetic foot who underwent surgical treatment. Results: Of the respondents $55 \%$ were female. Aged 38 to $93(65.08 \%)$ years. With income of up to two minimum wages (87.5\%), coming from Empress (32.5\%). Risk factors for amputation: $65 \%$ were smokers, $42.5 \%$ alcoholics and $65 \%$ had a family history of diabetes mellitus. As for comorbidities $57.5 \%$ had systemic arterial hypertension, $52.5 \%$ dyslipidemia and $27.5 \%$ coronary disease. About care and guidance: $32.5 \%$ were not in the habit of walking barefoot, $72.5 \%$ never had their feet examined by a healthcare professional and $87.5 \%$ never received care guidance by a healthcare professional. Conclusion: Most factors contributing to diabetic foot amputation can be prevented or controlled with preventive measures, such as simple care and self-care guidance, so as to corroborate the onset of diabetic foot and / or complications, especially amputations.
\end{abstract}

Key words: Diabetic foot, Risk factors, Amputation.

1Universidade Federal do Maranhão (UFMA), Imperatriz-Maranhão. *E-mail: janeidepereira2012@gmail.com 


\section{RESUMEN}

Objetivo: identificar los factores de riesgo que contribuyeron a la amputación mayor y menor en pacientes con pie diabético en el Hospital Municipal de Imperatriz. Métodos: Este es un estudio analítico de enfoque cuantitativo. Se utilizó un cuestionario que contenía variables sociodemográficas, aspectos socioeconómicos y aspectos clínicos. La muestra consistió en 40 pacientes con pie diabético que se sometieron a tratamiento quirúrgico. Resultados: De los encuestados, el 55\% eran mujeres. De 38 a 93 años $(65.08 \%)$ años. Con ingresos de hasta dos salarios mínimos (87.5\%), provenientes de Empress (32.5\%). Factores de riesgo de amputación: el $65 \%$ eran fumadores, el $42,5 \%$ alcohólicos y el $65 \%$ tenían antecedentes familiares de diabetes mellitus. En cuanto a las comorbilidades, el 57,5\% tenía hipertensión arterial sistémica, 52,5\% de dislipidemia y $27,5 \%$ de enfermedad coronaria. Acerca de la atención y la orientación: el $32.5 \%$ no tenía la costumbre de caminar descalzo, el $72.5 \%$ nunca tuvo los pies examinados por un profesional de la salud y el $87.5 \%$ nunca recibió orientación de la atención por parte de un profesional de la salud. Conclusión: La mayoría de los factores que contribuyen a la amputación del pie diabético pueden prevenirse o controlarse con medidas preventivas, como la atención simple y la guía de autocuidado, para corroborar la aparición del pie diabético y / o complicaciones, especialmente amputaciones.

Palabras clave: Pie diabético, Factores de riesgo, Amputación.

\section{INTRODUÇÃO}

O diabetes mellitus (DM) é um grande problema de saúde pública, pois é um distúrbio crônico com elevadas taxas de morbimortalidade, que afeta grande parte da população, e tem como causa fatores hereditários e ambientais, sendo um problema de saúde comum na população brasileira e sua prevalência alcançou as marcas de 6,2\% (Pesquisa Nacional de Saúde 2013) e 6,9\% vem aumentando nos últimos anos (BRASIL MS, 2016).

O pé diabético é uma das complicações crônicas mais frequentes do Diabetes Mellitus. Caracteriza-se pela presença de lesões nos pés em decorrência de alterações vasculares periféricas e/ou neurológicas peculiares do DM, constituindo-se pela tríade: neuropatia, doença vascular periférica e infecção. Se este agravo não for reconhecido precocemente, pode evoluir para gangrena e até mesmo amputação do membro (SANTOS ICRV, et al. 2011).

Muitas pessoas que são diagnosticadas com diabetes mellitus, têm risco para desenvolvimento de uma úlcera de pé estimada em 15\%. Com base em um estudo recente, a variações de incidência baseadas na população de $1,0 \%$ para $4,1 \%$, e a prevalência varia de $4 \%$ a $10 \%$, o que sugere que a incidência ao longo da vida será em torno de $25 \%$ para úlceras em extremidade inferior. O risco de ocorrerem tais amputações é 15 vezes maior em portadores de pé diabético (BONA SF, et al. 2010).

Em um estudo, é ressaltado que o pé diabético vem sendo uma das principais causas de amputação, porém, o mesmo pode ser prevenido, se houver identificação precoce do grau de risco para desenvolver alguma úlcera, sendo necessárias a adição de medidas preconizadas pelo Grupo de Trabalho Internacional Sobre Pé Diabético (VIDAL L, 2009).

O pé diabético, uma das principais causas de amputação, pode ser prevenido através da identificação precoce do grau de risco em desenvolver úlcera no pé e adoção de medidas, preconizadas pelo GTIPD (Grupo de Trabalho Internacional Sobre Pé Diabético), para evitar ou retardar a ulceração no pé (VIDAL L, 2009).

Fatores de risco como hiperglicemia prolongada, longo tempo de doença, dislipidemia, neuropatia, doença vascular periférica, presença de lesões ulcerativas e maus hábitos como tabagismo e uso de bebida alcoólica predispõem para amputações maiores ou transtibiais ou amputações menores ou transmetatarsianas (SPOLETT GR, 1998 apud GAMBA MA, et al. 2004).

A amputação maior ou menor de pé diabético no Brasil é uma conduta médica bastante abordado em tratamento de pacientes diabéticos e acredita-se que fatores como: idade, condições socioeconômicas, 
comorbidades, tempo com a patologia diabetes, uso de calçado inadequado, nível de educação, frequência de realização do curativo, hábitos de vida como tabagismo e etilismo influenciam em amputações maior ou menor do pé diabético (BRASIL MS, 2016).

Dentre os locais que surgem as lesões, os mais comuns são os dedos, que ocorrem devido as pressões externas que são elevadas, causadas pela atrofia da musculatura. As lesões também podem surgir nos sulcos interdigitais, devido a ocorrência de fissuras e pequenos cortes, o que proporciona a colonização por fungos na pele (CONSENSO INTERNACIONAL SOBRE O PÉ DIABÉTICO, 2001).

A neuropatia periférica é a complicação tardia mais comumente observada nos membros inferiores e constitui a principal causa relacionada ao aparecimento de úlceras nos pés e dois terços desses pacientes submetidos a amputação de um dos membros inferiores morrem em cinco anos, devido à restrição ao leito, acompanhamento psicológico inadequado e alimentação descontrolada (PHILBIN TM, LEYES M e SFERRA JJ, 2016).

Independente da origem do paciente com pé diabético, sabe-se que nos programas de atenção básica de saúde, existem estratégias específicas que visam evitar, diminuir e combater prognósticos mais severos para com esses pacientes (SOUSA LSN, et al. 2011).

Segundo dados da Pesquisa Nacional de Saúde, cerca de 47\% dos pacientes diabéticos ressaltam que receberam assistência médica durante o ano, isso no setor da atenção primária. Em consultórios particulares ou clínicas privadas, cerca de $29 \%$ afirmaram receber tal atendimento. Dessa forma, esses números significam que o acesso desses pacientes aos serviços de saúde, público ou privado, não garantes uma assistência de qualidade, sendo então, não suficientes para a prevenção de complicações de DM (BRASIL MS, 2016).

O pé diabético se caracteriza com um grande desafio para os gestores do sistema de saúde, uma vez que sua ocorrência causa impacto socioeconômico e algumas ações podem possibilitar a redução desse impacto através de intervenções bem articuladas e direcionadas (CONSENSO INTERNACIONAL SOBRE PÉ DIABÉTICO, 2001).

Sendo o pé diabético uma das mais mutilantes complicações crônicas do DM e diante do impacto social e econômico observado em todo o mundo, justifica-se o interesse na pesquisa de sua prevalência para programar medidas de prevenção, ainda carente de publicações (INTERNATIONAL DIABETES FEDERATION, 2012).

Em Imperatriz verificamos um número significativo de pacientes que sofreram tratamento cirúrgico de amputação de pé diabético no HMI, dados estes confirmados pelo SUS no ano de 2013 (DATASUS, 2013). Então realizamos esta pesquisa buscando verificar os fatores de risco nesses pacientes relacionados com essas amputações.

Assim, o presente estudo teve como objetivo identificar os fatores de risco que contribuíram para essas amputações maiores e menores em pacientes com pé diabético, assim como descrever o perfil sócio econômico, caracterizar os aspectos clínicos iniciais desses pacientes que passaram por amputações e investigar o tipo de cuidado desenvolvido com os pés pelos pacientes.

\section{MÉTODOS}

Trabalho realizado através de um estudo, analítico e com abordagem quantitativa. A pesquisa foi realizada no Hospital Municipal de Imperatriz (HMI) que é uma esfera administrativa municipal que dispõe de 105 médicos e 513 outros funcionários. O hospital presta atendimentos ambulatoriais de média e de alta complexidade assim também como os atendimentos nas internações, urgência e emergência com atuação em traumatologia, ortopedia, clínica médica, clínica cirúrgica, neurologia, pediatria entre outras, contêm 365 leitos para atendimento em clínica geral, atendimentos de especialização cirúrgica e leitos de UTI. Faz atendimento da população local e de outras cidades do Maranhão, Pará e Tocantins (DATASUS, 2017). 
Os participantes da pesquisa foram pacientes portadores de pé diabético que sofreram amputações maiores e menores de membro inferior, sendo que essa classificação consta no segundo o Consenso Internacional Sobre o Pé Diabético ano 2001 (CONSENSO INTERNACIONAL SOBRE O PÉ DIABÉTICO, 2001).

Foram considerados elegíveis para esta pesquisa pacientes diabéticos que: aceitaram participar voluntariamente da pesquisa, dando anuência de sua participação através da assinatura do termo de consentimento livre esclarecido, em duas vias (uma para o pesquisador outra ficando com o participante), portadores da patologia diabetes tipo 1 e 2, que sofreram amputação de grau maior ou menor do membro inferior.

A amostra desse estudo foi no total de 40 pacientes com pé diabético que sofreram tratamento cirúrgico no período de outubro a novembro de 2017 no HMI. Os critérios de exclusão foram: pacientes que não aceitaram assinar o termo de consentimento, que estavam impossibilitados de participar dos testes para pé diabético e que não tinham disponibilidade de tempo para realizar todo o questionário aplicado.

A pesquisa foi realizada no período de outubro a novembro 2017, cuja coleta foi realizada a partir de visitas ao hospital, respeitando os horários de alimentação e de visita de familiares na unidade, na oportunidade foram abordados os participantes em uma breve explanação acerca dos objetivos e finalidade do estudo, em seguida eles eram convidados a participar da pesquisa em estudo respondendo o questionário e participando dos teste de sensibilidade do pé diabético.

O questionário utilizado possuía variáveis sociodemográficas, aspectos socioeconômicos e relacionados a patologia. As perguntas presentes no questionários eram: nome, idade, sexo, grau de escolaridade e renda mensal, se era etilista, tabagista, presença de comorbidades como: hipertensão arterial, doença cardiovascular e dislipidemia, presença de história familiar de diabetes mellitus, se já tinha apresentado úlceras nos pés, apresentava dor ao caminhar, se os pés já tinha sido examinado por algum profissional de saúde, se já tinha recebido orientação sobre cuidados com os pés antes da amputação, presença de habito de caminhar descalço.

Durante a coleta dos dados foi feita uma observação do local da amputação, exame de inspeção, palpação e uso de aparelhos como: monofilamento de $10 \mathrm{~g}$ para sensibilidade dos pés, diapasão $128 \mathrm{~Hz}$ para sensibilidade vibratória, pino ou palito para sensibilidade dolorosa, martelo para reflexo aquileu, e algodão para sensibilidade superficial do membro não amputado para pesquisar risco de amputação do outro membro. Foi realizado também busca de informações complementares ao prontuário do participante como: início do tratamento, frequência de realização de curativo, equipe envolvida no curativo, e informações acerca da amputação.

Para análise dos dados foi utilizado a planilha estatística do Exce ${ }^{\circledR}$ (Microsoft, versão 2013) e o programa Word ${ }^{\circledR}$ (Microsoft, versão 2013), sendo agrupados, ordenados, contabilizados e tabulados, e com auxílio do programa SPSS, versão 24, com teste Qui-quadrado e T-Student, com p menor ou igual a 0,05 e intervalo de confiança de $95 \%$. Este trabalho segue as recomendações éticas da resolução Resolução № 466, de 12 de dezembro de 2012, que dá o direcionamento para pesquisas com seres humanos, em que foi submetido ao Comitê de Ética e Pesquisa da Faculdade de Imperatriz, com o número do protocolo 024-1\2017.

\section{RESULTADOS E DISCUSSÃO}

Após análise dos dados verificamos que dos 40 pacientes entrevistados $55 \%$ foram de sexo feminino, com variação de idade de 38 a 93 anos e que a escolaridade dos entrevistados apresentou como resultado $50 \%$ não alfabetizados. Que a renda, $87,5 \%$ dos participantes estava entre menos de um salário até dois salários mínimos.

Constatou-se também o local de procedência tinha a presença de pacientes de 4 estados somando um total de 24 municípios. Desses municípios, 32,5\% era de imperatriz e a maioria 67,5\% de outros municípios (Tabela 1). 
Tabela 1 - Perfil sociodemográfico de pessoas com amputação em membro inferior, Imperatriz, MA, 2017.

\begin{tabular}{llcc}
\hline Variáveis & & $\mathbf{N}(\mathbf{4 0})$ & \% (100) \\
\hline \multirow{4}{*}{ Idade em Faixa Etária } & $30-39$ & 1 & 2,5 \\
& $40-49$ & 3 & 7,2 \\
& $50-59$ & 7 & 17,5 \\
& $60-69$ & 11 & 27,5 \\
& $70-79$ & 14 & 35 \\
& $80-89$ & 3 & 7,5 \\
\multirow{2}{*}{ Sexo } & $90-100$ & 1 & 2,5 \\
\hline \multirow{5}{*}{ Escolairdade } & Masculino & 18 & 45,0 \\
& Feminino & 22 & 55,0 \\
\hline \multirow{4}{*}{ Renda Mensal } & Não alfabetizado & 20 & 50,0 \\
& Fundamental incompleto & 9 & 22,5 \\
& Fundamental completo & 7 & 17,5 \\
& Médio completo & 2 & 5,0 \\
& Superior completo & 2 & 5,0 \\
\hline \multirow{2}{*}{ Procedência } & 0-1 Salário Mínimo & 11 & 27,5 \\
& 1-2 Salários Mínimo & 24 & 60,0 \\
\hline Total & 2 -3 Salários mínimo & 4 & 10 \\
\hline Fon & $>4$ Salários mínimo & 1 & 2,5 \\
\hline
\end{tabular}

Fonte: Gois JPS, Chaves ASC, 2019.

Acredita-se que com o avançar da idade é de se esperar o aparecimento de algumas patologias como diabetes e isso ficou caracterizado nessa pesquisa pela média da idade dos pacientes que está em torno dos 65 anos, ou seja, pessoas idosas que apresentam diabetes (JOICE PCG, 2011).

Santos et al., (2011) em sua pesquisa Prevalência e fatores associados a amputações por pé diabético observou que de 214 portadores de pé diabéticos no hospital estudado em seu trabalho $107(50 \%)$ foram submetidos à amputação de algum segmento dos membros inferiores sendo que a idade média dos pacientes foi de 65 anos (mediana $=65$ anos; $D P=11,6$ ) e o teste de associação entre as variáveis categóricas mostrou significância estatística $(p<0,05)$ para as variáveis: anos de estudo dos pacientes.

Dentre as complicações mais debilitantes do diabetes, do ponto de vista funcional para o idoso, o pé diabético se destaca como um agravo que irá interferir constantemente em suas funções e, se não tratado, evolui para amputação de membro, impossibilitando o indivíduo de realizar suas atividades de vida diária, tornando-se dependente de outros (JOYCE JCG, 2011).

Doenças como: doença arterial periférica, neuropatia periférica, ulceração do pé, ou amputação das extremidades inferiores, são duas vezes mais comuns em pessoas diabéticas quando comparadas com os não diabéticos e afeta $30 \%$ dos pacientes diabéticos com mais de 40 anos (SINGH N, ARMSTRONG DG e LIPSKY BA, 2015).

Com relação a escolaridade os resultados mostraram que $90 \%$ dos pacientes vão de não alfabetizados a fundamental incompleto, sendo que $50 \%$ desses são analfabetos. O controle do DM deve ser valorizado no acompanhamento e tratamento do paciente. Nesse sentido, destacasse a educação da pessoa com diabetes, como um aspecto fundamental do cuidado na obtenção do controle da doença e, assim, prevenir ou retardar o desencadeamento de complicações agudas e crônicas, ajudando-os na promoção da qualidade de vida (BARBOSA E CAMBOIM apud RODRIGUES et al.,2012). 
Com relação ao poder aquisitivo dos pacientes, $87,5 \%$ tinha como renda até dois salários mínimos, demostrando que a maior parte dos entrevistados nessa pesquisa apresentaram um poder aquisitivo baixo, sendo que pacientes diabéticos necessitam de uma alimentação que exige um gasto maior, pois os mesmos tem despesa com medições e produtos para autocuidado dos pés, somando um gasto que pode ir além da renda do paciente (SINGH N, ARMSTRONG DG e LIPSKY BA, 2015).

Santos ICRV, et al, (2011) em sua pesquisa Prevalência e fatores associados a amputações por pé diabético observou que a renda dos pacientes foi de até um salário mínimo o que correspondeu a 92,5\% dos pacientes do estudo sofreu amputações. Globalmente o pé diabético continua a ser um grande problema social, econômico e médico, afetando $15 \%$ do total de diabéticos e constitui a maior causa de amputação nãotraumática na maioria dos países ocidentais, sendo a taxa de amputação nos diabéticos 15 vezes maior em comparação com a população não diabética (LEITE FEOPC, 2009; LEITE FEOPC, 2010).

A Tabela 2 mostra os fatores de risco para amputação de pé diabético avaliados no questionário que foram: $65 \%$ dos amputados eram tabagistas, $42,5 \%$ etilista e $65 \%$ tinha história de DM na família. Com relação a comorbidades, verificamos a presença de Hipertensão Arterial Sistêmica em 57,5\% dos entrevistados, dislipidemia em $52,5 \%$ e doença coronariana $27,5 \%$.

Tabela 2 - Fatores de risco e comorbidades identificadas, Imperatriz, MA, 2017.

\begin{tabular}{lccc}
\hline Fatores de risco e cormobidades & & $\mathbf{N}(\mathbf{4 0})$ & \% (100) \\
\hline Tabagismo & Sim & 26 & 65,0 \\
& Não & 14 & 35,0 \\
Etilismo & Sim & 17 & 42,5 \\
& Não & 23 & 57,5 \\
Hipertensão Arterial Sistêmica & Sim & 23 & 57,5 \\
& Não & 17 & 42,5 \\
Dislipidemia & Sim & 21 & 52,5 \\
& Não & 19 & 47,5 \\
Doença Cardiovascular & Sim & 11 & 27,5 \\
História Familiar de Diabetes & Não & 29 & 72,5 \\
Mellitus & Sim & 26 & 65,0 \\
\hline Total & Não & 14 & 35,0 \\
\hline
\end{tabular}

Fonte: Gois JPS, Chaves ASC, 2019.

Dentre as comorbidades em estudo verificamos que a Hipertensão Arterial Sistêmica é a comorbidade que mais aparece como fator de risco na pesquisa e que a doença coronariana nesse estudo foi o fator de risco em menor número. Os fatores de risco para o desenvolvimento de pé diabético são: idade, tipo e tempo de diagnóstico, controle inadequado da glicemia, tabagismo, alcoolismo, obesidade, hipertensão e falta de hábitos higiênicos e cuidado local (THOMAZELLI FCS, MACHADO CB e DOLCAN SK, 2015).

Na Tabela 3 mostra resultados sobre cuidados e orientações com os pés, sendo que teve seus pés examinados por um profissional, $27,5 \%$, recebeu orientações sobre cuidados com os pés antes da amputação, 12,5\%, tem hábito de caminhar descalço, 32,5\%.

Tabela 3 - Cuidados e orientações recebidas sobre cuidados aos pés, Imperatriz, MA, 2017.

\begin{tabular}{lccc}
\hline Itens questionados & & $\mathbf{n ( 4 0 )}$ & \%(100) \\
\hline Já teve seus pés examinados por um & Sim & 11 & 27,5 \\
profissional? & Não & 29 & 72,5 \\
Já recebeu orientações sobre cuidados com & Sim & 5 & 12,5 \\
os pés antes da amputação? & Não & 35 & 87,5 \\
Tem hábito de caminhar descalço? & Sim & 13 & 32,5 \\
& Não & 27 & 67,5 \\
\hline Total & & $\mathbf{4 0}$ & $\mathbf{1 0 0}$ \\
\hline
\end{tabular}

Fonte: Gois JPS, Chaves ASC, 2019. 
De acordo com os resultados verificamos que $67,5 \%$ dos pacientes são provenientes de municípios que tem cobertura de atenção básica e que como resultado a maioria não teve seus pés examinados antes de complicações que evoluiu a amputação e não receberam orientações suficientes sobre os cuidados com os pés. Diante disso, o profissional precisa direcionar sua atenção para as alterações decorrentes do pé diabético, assim como os fatores de riscos que podem vir a ser modificados, sendo assim, será possível evitar úlceras, infecções e por consequência a amputação. Pois, através de técnicas simples de autocuidado com os pés, é possível prevenir um número significativo dessas amputações (APELQVISTJ, et al, 2007).

O Consenso Internacional sugere a adoção do sistema de classificação de risco do pé diabético (GITPD) que além de identificar o grau de risco também orienta as medidas terapêuticas necessárias que o profissional deve tomar seguindo a seguinte classificação: paciente sem neuropatia, examinar o pé uma vez por ano, com neuropatia uma vez a cada 6 meses, com neuropatia, sinais de doença venosa periférica uma vez a cada 3 meses e/ou presença de deformidades, amputação, ulceração prévia uma vez a cada 1 a 3 meses (CONSENSO INTERNACIONAL SOBRE PÉ DIABÉTICO, 2001).

Tabela 4 - Classificação das amputações identificadas segundo local de realização, Imperatriz, MA, 2017.

\begin{tabular}{|c|c|c|}
\hline Tipo de Amputação & $\mathrm{N}(40)$ & $\%(\mathbf{1 0 0})$ \\
\hline \multicolumn{3}{|l|}{ Amputações Menores } \\
\hline 3o Pododáctilo esquerdo & 4 & 10,0 \\
\hline Hálux esquerdo & 3 & 7,5 \\
\hline Hálux e $2^{\circ}$ pododáctilo direito & 2 & 5,0 \\
\hline Calcâneo esquerdo & 2 & 5,0 \\
\hline 2º Pododáctilo esquerdo & 3 & 7,5 \\
\hline 2º Pododáctilo direito & 3 & 7,5 \\
\hline 5ํPododáctilo direito & 3 & 7,5 \\
\hline $2^{\circ}, 3^{\circ}$ e $4^{\circ}$ pododáctilo direito & 3 & 7,5 \\
\hline Calcâneo direito & 1 & 2,5 \\
\hline 1ํPododáctilo esquerdo & 3 & 7,5 \\
\hline $1^{\circ}$ e $2^{\circ}$ Pododáctilo esquerdo & 2 & 5,0 \\
\hline Total amputações menores & 29 & 72,5 \\
\hline \multicolumn{3}{|l|}{ Amputações Maiores } \\
\hline Tíbia direita & 3 & 7,5 \\
\hline Coxa direita & 4 & 10,0 \\
\hline Pé esquerdo & 2 & 5,0 \\
\hline Pé direito & 2 & 5,0 \\
\hline Total amputações maiores & 11 & 27,5 \\
\hline Total & 40 & 100,0 \\
\hline
\end{tabular}

Fonte: Gois JPS, Chaves ASC, 2019.

Na Tabela 4 verificamos que o número de amputações de nível menor nos pacientes em estudo foi $72,5 \%$, sendo que essas amputações foram de um pododáctilo chegando à parte do pé como o calcâneo.

Em um estudo foi demonstrado que fatores como a hiperglicemia prolongada, longo tempo de doença, dislipidemia, neuropatia, doença vascular periférica, presença de lesões ulcerativas e maus hábitos como tabagismo e uso de bebida alcoólica predispõem para amputações maiores ou transtibiais ou amputações menores ou transmetatarsianas (SPOLETT GR, 1998 apud GAMBA MA, et al, 2004).

Em muitos países a incidência de amputações de membros inferiores tem diminuído nas últimas duas décadas como resultado de ações preventivas organizadas, avanços nas técnicas cirúrgicas e cuidado multidisciplinar do usuário. Entretanto ainda existem exemplos em que a incidência continua inalterada a despeito das medidas específicas tomadas (POLJIĖANIN T, et al, 2005). 
Considerando a amostra apresentada, observa-se que a distribuição de faixa etária não apresentou diferenças de valores significativos.

Tabela 5 - Relação entre o número de amputação e idade, Imperatriz, MA, 2019.

\begin{tabular}{|c|c|c|c|c|c|c|c|c|c|}
\hline \multirow{2}{*}{ Amputação } & \multicolumn{7}{|c|}{ Idade em Faixa etária } & \multirow[b]{2}{*}{ Total } & \multirow[b]{2}{*}{$p$} \\
\hline & $30-39$ & $40-49$ & $50-59$ & $60-69$ & $70-79$ & $80-89$ & $90-99$ & & \\
\hline 3 Pododáctilo esquerdo & 0 & 0 & 0 & 2 & 2 & 0 & 0 & 4 & \\
\hline Hálux esquerdo & 0 & 0 & 0 & 2 & 1 & 0 & 0 & 3 & \\
\hline Hálux e $2^{\circ}$ pododáctilo direito & 0 & 0 & 1 & 0 & 1 & 0 & 0 & 2 & \\
\hline Pé direito & 0 & 0 & 1 & 0 & 1 & 0 & 0 & 2 & \\
\hline 2 Pododáctilo esquerdo & 0 & 0 & 0 & 0 & 2 & 1 & 0 & 3 & \\
\hline Tíbia direita & 0 & 0 & 1 & 2 & 0 & 0 & 0 & 3 & \\
\hline 5 Pododáctilo direito & 0 & 1 & 0 & 2 & 0 & 0 & 0 & 3 & \\
\hline $2^{\circ}, 3^{\circ}$ e $4^{\circ}$ pododáctilo direito & 0 & 1 & 0 & 1 & 1 & 0 & 0 & 3 & 0,403 \\
\hline Calcâneo direito & 0 & 0 & 0 & 0 & 1 & 0 & 0 & 1 & 0,400 \\
\hline 1ㅇ Pododáctilo esquerdo & 1 & 1 & 0 & 0 & 1 & 0 & 0 & 3 & \\
\hline $1^{\circ}$ e $2^{\circ}$ Pododáctilo esquerdo & 0 & 0 & 1 & 1 & 0 & 0 & 0 & 2 & \\
\hline 2ํㅜ Pododáctilo direito & 0 & 0 & 1 & 1 & 0 & 1 & 0 & 3 & \\
\hline Coxa direita & 0 & 0 & 2 & 0 & 2 & 0 & 0 & 4 & \\
\hline Pé esquerdo & 0 & 0 & 0 & 0 & 2 & 0 & 0 & 2 & \\
\hline Calcâneo esquerdo & 0 & 0 & 0 & 0 & 0 & 1 & 1 & 2 & \\
\hline Total & 1 & 3 & 7 & 11 & 14 & 3 & 1 & 40 & \\
\hline
\end{tabular}

Fonte: Gois JPS, Chaves ASC, 2019.

\section{CONCLUSÃO}

Concluímos que a maior parte dos pacientes deste estudo apresentam uma renda baixa, fator que pode dificultar o acesso a compra de algumas medicações e uma alimentação mais adequada para seu estado patológico. Verificamos que todos os pacientes do estudo são de municípios que têm cobertura de atenção básica, que a maioria dos pacientes apresentava um baixo nível de instrução, e que o baixo nível de escolaridade e o analfabetismo possam complicar o entendimento do paciente sobre o autocuidado do pé diabético e/ou aparecimento dessa complicação, podendo colaborar para uma amputação maior ou menor do membro.

\section{REFERÊNCIAS}

1. APELQVIST J, et al. Practical guidelines on the management and prevention of the diabetic foot. Based upon the International Consensus on the Diabetic Foot (2007). Prepared by the International Working Group on the Diabetic Foot. Diabetes Metab. Res. Rev., v. 24, p. S181-S187, 2008.

2. BARBOSA SA e CAMBOIM FE. Diabetes mellitus: cuidados de enfermagem para controle e prevenção de complicações. Disponível em: http://temasemsaude.com/wpcontent/uploads/2016/09/16324.pdf Acesso em 14 de novembro de 2017.

3. BRASIL Ministério da Saúde. Secretaria de Atenção à Saúde. Departamento de Atenção Básica. Manual do pé diabético: estratégias para o cuidado da pessoa com doença crônica / Ministério da Saúde, Secretaria de Atenção à Saúde, Departamento de Atenção Básica. - Brasília: Ministério da Saúde, 2016.

4. BRASIL Ministério da Saúde. Secretaria de Atenção à Saúde. Departamento de Atenção Básica. Estratégias para o cuidado da pessoa com doença crônica: diabetes mellitus. Brasília: Ministério da Saúde, 2013.

5. Ministério do Planejamento, Orçamento e Gestão. Instituto Brasileiro de Geografia e Estatística. Pesquisa Nacional de Saúde 2013: Percepção do estado de saúde, estilos de vida e doenças crônicas. Brasília: Rio de Janeiro, 2014a.

6. Ministério da Saúde. Secretaria de Vigilância em Saúde. Vigitel Brasil 2013: vigilância de fatores de risco e proteção para doenças crônicas por inquérito telefônico. Brasília: Ministério da Saúde, 2014b.

7. Ministério da Saúde. DATASUS, Disponível em: http://tabnet.datasus.gov.br/cgi/tabcgi.exe?hiperdia/cnv/hdMA.def> acessado em: 11 nov. 2017. 
8. BONA SF, BARBOSA MAR, FERRAZ CLH e GUARITA LKS. Prevalência do pé diabético nos pacientes atendidos na emergência de um hospital público terciário de Fortaleza, Bras. Clínica Médica. 2010:8:1-5.

9. CONSENSO INTERNACIONAL SOBRE PÉ DIABÉTICO. Grupo de Trabalho Internacional sobre o Pé Diabético. Secretaria de Estado de Saúde do Distrito Federal. Direção: Pedrosa, H.C., Brasília, 2001. 20p.

10. GAMBA MA, et al. Amputações de extremidades inferiores por diabetes mellitus: estudo caso-controle. Revista Saúde Pública, v. 38, n.3, p. 44 - 48, Junho 2004.

11. INTERNATIONAL DIABETES FEDERATION. Clinical Guidelines Task Force. Global Guideline for Type 2 Diabetes. 2012. Disponível em: http://www.idf.org/guideline-type-2-diabetes>. Acesso em: 8 mar 2017.

12. JOYCE PCG. Avaliação de risco para pé diabético em idosos portadores de diabetes mellitus tipo 2. Dissertação de Mestrado em Enfermagem do Programa de Pós-Graduação da Escola de Enfermagem da Universidade Federal de Minas Gerais. Belo Horizonte, 08 de Julho de 2011.

13. LEITE FEOPC. Pé Diabético, tese de mestrado em medicina. 2009/2010.

14. LOPES ASL, et al. Abordagem multidisciplinar do paciente diabético com infecção nos pés: prevenção de amputações maiores. 2010.

15. PINZUR, MS, SLOVENKAI MP e TREPMAN E.: Diabetes Committee of American Orthopaedic Foot and Ankle Society. Guidelines for diabetic foot care: recommendations endorsed by the. 2005.

16. PHILBIN TM, LEYES M, e SFERRA JJ . Orthotic and prosthetic devices in partial foot amputations. Foot Ankle Clin 2016: 215-28.

17. POLJIĖANIN T, et al. Draft program of prevention of diabetic foot Development and lower extremity amputation in Persons with diabetes mellitus. Diabetol Croat. 2005; 34(2):43-9.

18. SANTOS ICRV, et al. Pé diabético:apresentação clínica e relação com o atendimento na atenção básica. Rene. Fortaleza. 2011. 12 (2): 393-400.

19. SOUSA LSN, et al. Cuidados ao Paciente com pé diabético: Pesquisa Bibliográfica, 2011.

20. SINGH N, ARMSTRONG D.G e LIPSKY B.A. Preventing foot ulcers in patients with diabetes. Clinician's Corner 2015. http://jama.jamanetwork.com/ by Allon Dubler on 02/27/2015.

21. SPOLETT GR. Preventing amputations in the diabetic population. Nursing Clinic of North America, Boston, v. $33, \mathrm{n}$. 4, p. 629 -641, Dec. 1998.

22. THOMAZELLI FCS, MACHADO CB e DOLÇAN SK. Risk of diabetic foot in an interdisciplinary outpatient clinic of diabetes. Revista da AMRIGS, Porto Alegre, 59 (1): 10-14, jan.-mar. 2015.

23. VIDAL L. Avaliação do sistema de classificação de risco do pé, proposto pelogrupo de trabalho internacional sobre o pé diabético, Hospital da PoliciaMilitar de Minas Gerais, 2002-2007. Belo Horizonte: 2009. 